\title{
Insights on Spectrum Sharing in Heterogeneous Networks with Small Cells
}

\author{
Bruno C. Silva, Sofia C. Sousa, Emanuel Teixeira, Fernando J. Velez \\ Instituto das Telecomunicações \\ Universidade da Beira Interior, DEM, Faculdade de Engenharia \\ 6201-001 Covilhã, Portugal \\ bruno.cruz.silva@ubi.pt, sofia.sousa@lx.it.pt, emanuelt@ubi.pt, fjv@ubi.pt
}

\begin{abstract}
This work explores the viability of 5G New Radio spectrum sharing in Ultra High Frequency (UHF), Super High Frequency (SHF) and millimetre wavebands(mmWaves) in outdoor environments. In the mmWaves the linear cellular topology is considered while in the UHF/SHF bands cells with hexagonal shape are assumed. Performance evaluation includes the study of the behaviour of PHY and supported throughput for 2.6, 3.5, 28, 38, 60 and $73 \mathrm{GHz}$. While the two-slope model is considered for the 2.6 and $3.5 \mathrm{GHz}$ frequency bands, the modified Friis propagation model, with shadow fading, and different values for the standard deviation, is the considered in the millimetre wavebands. With sharing, lower system capacity is supported. We clearly observe that, for coverage distances up to circa $100 \mathrm{~m}$, the supported throughput is higher at the millimetre wavebands, mainly due to the reduction that characterizes the application of the two-slope propagation model at the UHF/SHF bands.
\end{abstract}

Keywords-LTE-Advanced, ITU-R propagation model, System capacity, Spectrum sharing, HetNet

\section{INTRODUCTION}

Low availability of land for base stations installations, intercell interference, and costly infrastructure are obstacles to increasing network coverage and capacity. These Heterogeneous Network (HetNet) scenarios towards 5G, small cell (SC) based Cloud radio Access Networks (C-RAN) [1] are an excellent alternative that offers enhanced interference control, access control, and manageability. In C-RAN, baseband processing is centralized and shared among sites in a virtualized baseband unit (BBU) pool. This means that it is able to adapt to non- uniform traffic, for example traffic in the office during the day and residential traffic during the night, and utilizes the resources, i.e., base stations, more efficiently.

While Rel-14 focused solely on 5G study items, the normative work for the first phase of $5 \mathrm{G}$ system specification has started within Rel-15 targeting freeze date of September 2018 , while the so-called non-standalone (NSA) "5G version" targets its completion half year earlier. The 3GPP Rel-15 features, covering LTE enhancements and towards $5 \mathrm{G}$ aspects, are shown in Fig. 1. The first phase of the 5G systems covering the most immediate set of use cases envisioned for $5 \mathrm{G}$ is going to be completed within Rel-15, including enhanced Mobile Broadband (eMBB), and some Ultra-Reliable Low latency Communications (URLLC) aspects, while the second phase targets Rel-16. The First set of work-items for 5G phase 1 includes 5G Dual connectivity and LTE Connectivity to 5G.

This work has been partially supported and funded by COST CA 15104 UID/EEA/500008/2013, CONQUEST (CMU/ECE/0030/2017) and ORCIP.

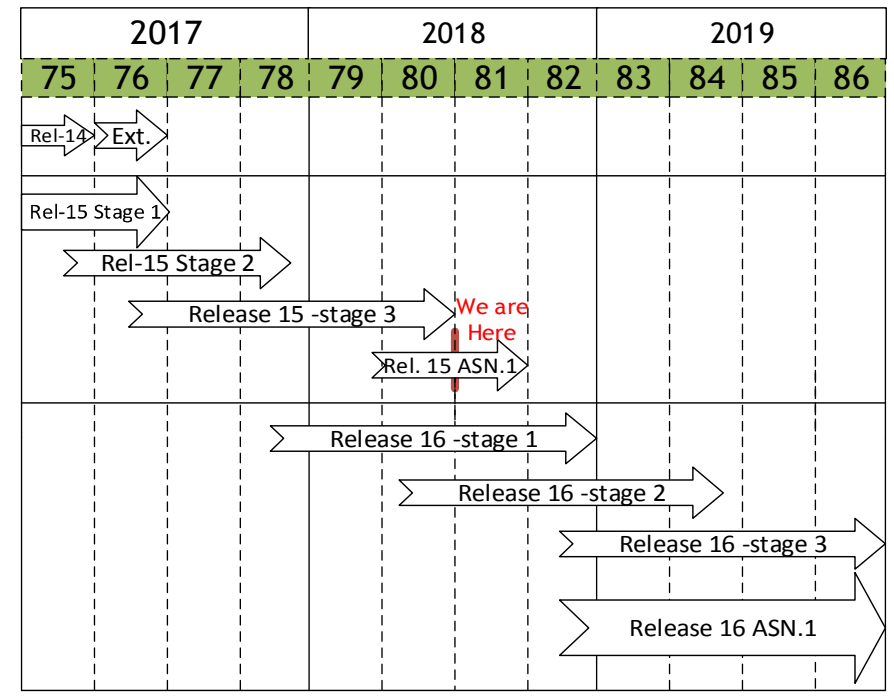

Fig. 1. 3GPP timeline.

Core Network (5G-CN), [2]. In the first phase of 5G system specification, the radio access called New Radio (NR) should cover the features to serve eMBB and URLLC use cases using the frequency ranges of up to $52.6 \mathrm{GHz}$. One of the key aspects for NR in Rel-15 is to allow forward compatibility for smooth introduction of the features that will be brought within Rel-16 along with $5 \mathrm{G}$ phase 2 . The approval of a study of $5 \mathrm{G}$ NR in unlicensed spectrum includes both an 'anchor' in licensed spectrum (the 5G licensed assisted access, LAA, version) and stand-alone in unlicensed spectrum (the 5G MulteFire version), i.e., within NR Spectrum Sharing, NR in unlicensed will become the $5 \mathrm{G}$ evolution path for both LAA and Multefire.

The spectrum scarcity nowadays, and the policy of spectrum licensing, whereby wireless systems get exclusive access to spectrum, potentially leads to highly inefficient use of spectrum. Large portion of the assigned spectrum are used sporadically as other bands have an increase in spectrum demand. Unlicensed and licensed shared spectrum have a set of possible advantages [3], [4], which can potentially lead to greater spectral efficiency than exclusive access. Optimization of the access using shared bands allows the assignment of the underutilized spectrum resources, where spectrum can be shared between license holders and secondary users. According to [5], dynamic spectrum sharing can be done through dynamic spectrum allocation, open sharing or hierarchical access mode. 3GPP 
Release 13-14 have adopted the LAA technology to deploy LTE-A networks on the $5 \mathrm{GHz}$ unlicensed bands [6], [7]. LAA has adopted CA as a mandatory function, and a licensed carrier must coexist with unlicensed carrier. SCs are the adequate candidate to accomplish the regulation in terms of limitation on the maximum transmitter power. Sharing between primary and secondary users is most useful when primary system has been give exclusive rights through licensing, as there are generally times and/or locations where other devices could transmit in this spectrum without causing harmful interference. In the context of spectrum sharing within Rel-15, these NR access technologies include i) LTE-Unlicensed/ LAA, ii) LTE-WLAN Aggregation (LWA), iii) Multefire (an LTE-based technology for SCs operating solely in unlicensed spectrum) and iv) Citizen Broadband Radio Service/ Licensed Shared Access (LSA).

In a simplistic approach, a scenario with "no sharing", where each mobile operator (MO) has dedicated licensed spectrum for SCs, could be considered. In this framework, MOs are free to optimize throughput and packet loss ratio with respective frequency reuse. However, as there are limitations in the availability of dedicated spectrum for each operator, this work considers a second case of sharing without coordination, which means that each operator adopts the same frequency reuse strategy as in the first scenario. Spectrum sharing access assumes that two or more MOs have dedicated spectrum for macro cellular layer while SCs will share the access to spectrum in an opportunistic manner. In this research, we address a very simple sharing scenario, in the SC layer, where we simply start adding a/various co-channel cells, from a different operator, over the cellular topology from the initial operator.

The aim of the work is to compare the system capacity, measured by the supported throughput, for cells of few hundred meters coverage in scenarios with simple frequency sharing configurations. The frequency bands in the SC layer are the 2.6 or $3.5 \mathrm{GHz}$, and $28,38,60$ or $73 \mathrm{GHz}$.

The remainder of the paper is organized as follows. Section II presents the scenario and topology of the sharing framework for Ultra High Frequency (UHF), Super High Frequency (SHF) and millimetre wavebands (mmWaves), and address the formulation for the carrier-to-interference ratio, $C / I$, that allows for modelling the reuse topologies and the underlying frequency sharing topologies, with simple assumptions. In section III, the performance evaluation considers the mapping between the carrier-to-noise-plus-interference ratio (CNIR) and the physical throughput, to obtain the supported throughput through an implicit function formulation. The analysis of the results facilitate to understand the frequency reuse trade-off in such scenarios and comparing the use of frequency sharing among different bands. Finally, conclusions are drawn in Section IV.

\section{SHARING FRAMEWORK}

\section{A. Scenario and topology}

In scenarios with spectrum sharing it is expected to see small cells from different operators placed close to each other. The challenge is to evaluate the pros- and cons- of dedicated spectrum compared to shared spectrum. In this initial effort, we decided to address a SC sharing scenario where we simply start adding a/various co-channel cells from a different operator positioned in the six corners of the central small cell from the initial operator, as shown in Fig. 2.

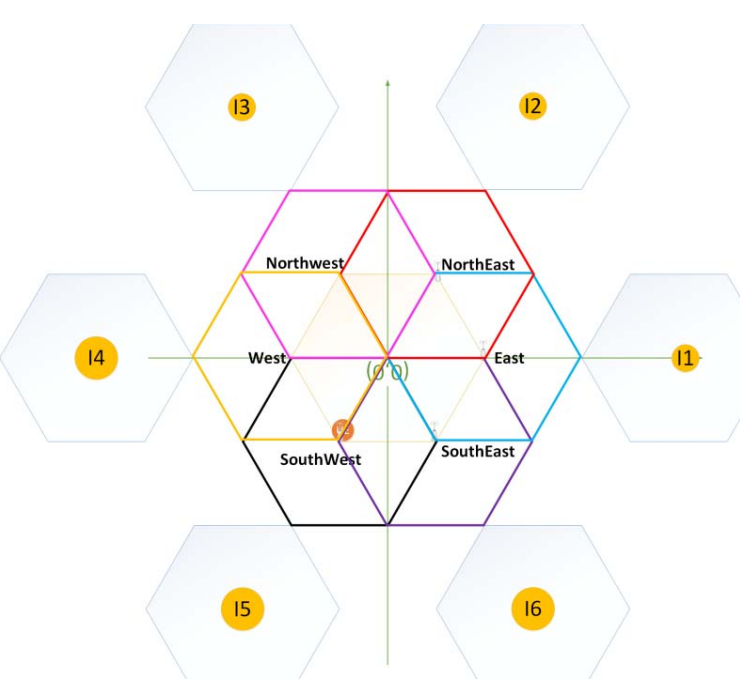

Fig. 2. Definition of the six different interferers for the sharing framework, where different cases are superimposed.

From a detailed analysis of the variation of the supported throughput with the coverage and reuse distances, for different values of the Channel Quality Indicator (CQI) and given ITU-R propagation models, an evaluation of the possible range for the reuse factor is performed for the downlink (DL) of LTE-A.

By considering CQI and reference carrier-to-noise-plusinterference ratio (CNIR) requirements recommended by 3GPP, DL peak bit rates along with the Transport Block Size assumed for single stream and bandwidths of 10 and $20 \mathrm{MHz}, \mathrm{PHY}$ and supported throughputs are analysed. Whereas the $10 \mathrm{MHz}$ bandwidth is considered for mobile systems operating in the $2.6 \mathrm{GHz}$ and $3.5 \mathrm{GHz}$ bands, $20 \mathrm{MHz}$ bandwidth is considered at the mmWaves $(28,38,60$ and $73 \mathrm{GHz})$, as in [8].

In the UHF and SHF bands, six scenarios are considered, East, Northeast, Southwest, Southeast, Northwest and West. The interference that outcomes from an interferer placed at the east corner is equal to the one form the northwest corner (represented in blue and pink, respectively, Fig. 2). Also the interference that outcomes from an interferer placed at the west corner is equal to southeast corner (represented in yellow and purple, respectively). For this reason, four out of six cases are going to be analysed, i.e., southwest, southeast, east and northeast. Fig.4 shows the position of the six considered interferers.

The following cases are superimposed in Fig. 2. The interferer ( $\mathrm{SC}$ from $\mathrm{MO} \# 2$ ) is in the i) Southeast of the central cell, ii) left hand side (Southwest) of the central cell, iii) East and iv) Northeast, causing interference in the central cell, on top of the other six co-channel cells at the first ring of interference (from MO \#1). These four cases are represented in i) purple, ii) black (this is the worst-case), iii) blue and iv) red, respectively;

The UHF/SHF frequency bands considered for the small cells, by now, are $2.6 \mathrm{GHz}$ and $3.5 \mathrm{GHz}$. For every case, we present the variation of CNIR and PHY throughput with the distance, $d$, for different cells sizes. The distance from the user to the eNB, $d$, verifies $0 \leq d \leq R$ (e.g., $R=150 \mathrm{~m}$ ). Line-of-sight (LoS) propagation is assumed, and preliminary results are obtained for a bandwidth $B W=10 \mathrm{MHz}$.

In order to study the variation of the CNIR and supported throughput with the coverage distance, $R$, the supported throughput is computed through the implicit function analytical 
formulation from [9]. The supported throughput is compared for $2.6 \mathrm{GHz}$ (UHF), 3.5 GHz (SHF) , 28, 38, 60 and $73 \mathrm{GHz}$ (mmWaves) for a bandwidth $B W=20 \mathrm{MHz}$.

Fig. 3 presents the hexagonal-shaped interference topology for the UHF/SHF frequency bands, where eNBs from the six cochannel cells form MO \#1 cause interference in the user equipment at the edge of the central cell (downlink), and the eNB from MO \#2 is an additional source of interference. In the depiction to compute the variation of the PHY throughput throughout the cell, the user moves from the centre to the cell edge through a straight line. In this case, in the formulation for $C / I, R$ (the distance from the eNB to the user equipment in the cell edge, in the worst-case) is replaced by $d$, the distance from the eNB (centre of the cell) to the cell edge, so $0 \leq d \leq R$. This replacement is because the user is not on the cell edge anymore. Along this path, the user will get interference not only from the six co-channel cells but also from the additional interferer (MO \#2), in this case positioned at Northeast.

\section{B. Formulation}

Considering the distance between the user and the cochannel cell $\mathrm{F}$ represented as $d_{U F}$, or from the user to the cochannel cell $E$ represented as $d_{U E}$, the equation for the distance of the interferer eNB, using the shared frequency, is as follows:

$$
\begin{aligned}
& d_{U F}=\sqrt{\left(-\frac{d}{2}-3 R\right)^{2}+\left(-\frac{\sqrt{3}}{2} d\right)^{2}} \\
& d_{U E}=\sqrt{\left(-\frac{d}{2}+3 R\right)^{2}+\left(-\frac{\sqrt{3}}{2} d\right)^{2}}
\end{aligned}
$$

Note that, for the path considered for the user, the interference from cell $\mathrm{F}$ at the user equipment is equal to the interference from cell $\mathrm{B}$, while the interference form cell $\mathrm{E}$ is equal to the one from $\mathrm{C}$.

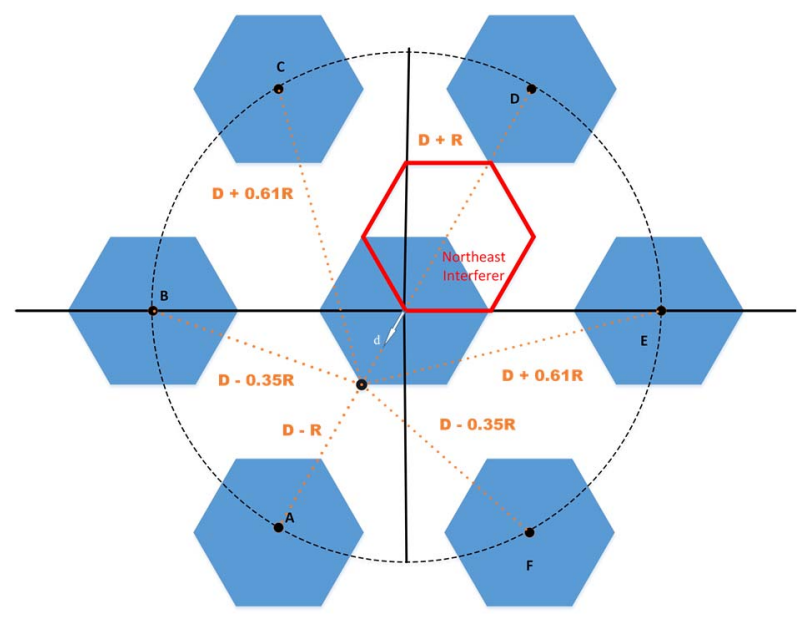

Fig. 3. Scenario in the UHF/SHF bands with $K=3$ and one $\mathrm{SC}$ interferer (Northeast).

The underlying equation for the carrier-to-interference ratio (without considering the interference from the cell that uses the shared band) is as follows:

$$
\frac{C}{I}=\frac{d^{-\gamma}}{2(d U F)^{-\gamma}+(D+d)^{-\gamma}+(D-d)^{-\gamma}+2(d U E)^{-\gamma}}
$$

where $D$ is the reuse distance.

To consider sharing corresponds to add $d_{I-\text { sharing }}{ }^{-\gamma}$ in the denominator, where $d_{I \text {-sharing }}$ is computed as follows:

- East Interferer:

$$
\sqrt{\left(R+\frac{d}{2}\right)^{2}+\left(\frac{\sqrt{3}}{2} d\right)^{2}}
$$

- Northeast Interferer:

$$
\sqrt{\left(\frac{R}{2}+\frac{d}{2}\right)^{2}+\left(\frac{\sqrt{3}}{2} d+\frac{\sqrt{3}}{2} R\right)^{2}}
$$

- Southwest Interferer:

$$
\sqrt{\frac{R}{\left(\frac{R}{2}+\frac{d}{2}\right)^{2}+\left(\frac{\sqrt{3}}{2} d-\frac{\sqrt{3}}{2} R\right)^{2}}}
$$

- Southeast Interferer:

The representation above considers the cell that uses the shared band and the user straight line path mentioned above.

In practice, one obtains the following equation for the carrier-to-interference ratio:

$$
\frac{C}{I}=\frac{d^{-\gamma}}{2 d_{U F}^{-\gamma}+(D+d)^{-\gamma}+(D-d)^{-\gamma}+2 d_{U E}^{-\gamma}+d_{I-\text { sharing }}^{-\gamma}}
$$

The values represented in (2) are obtained when we consider only the interference from co-channel cells, by replacing $R$ by $d$ (user equipment not placed on the cell edge anymore) in Fig. 3.

The propagation model and formulations that are applied in the UHF/SHF bands are proposed in [10]. In the mmWaves, we consider a linear topology, as in [8], but with an additional cell from the second mobile operator (and LTE as the air interface, as a simplification). The cell belonging to MO \#2 that uses the shared band is shown in Fig. 4 (green cell). We consider the linear cellular topology in an attempt to represent the worst-case of CNIR from a Manhattan grid topology, which are alike the worst-case bounds from a linear cellular topology. The interference geometry is shown in Fig. 4, with reuse pattern $K=2$, as described in [8], where the user moves horizontally from the centre to the edge of the cell.

In this topology, the equation for the carrier-to-interference ratio in the user equipment at a distance $d$ from the eNB (downlink) is as follows:

$$
\frac{C}{I}=\frac{d^{-\gamma}}{(D-d)^{-\gamma}+(D+d)^{-\gamma}+(R+d)^{-\gamma}}
$$

where $D=4 \cdot R$ in the specific topology from Fig. 4 . The interferer from MO \#2 is at the left hand side of the original central cell and the interferer coming from it is at distance $R+d$ from the user equipment (UE).

The propagation model and formulations that are applied in the mmWaves (mainly in LoS) are presented in [8].

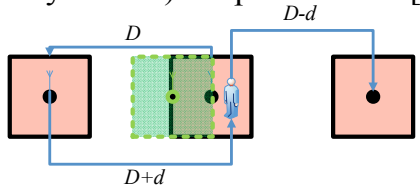

Fig. 4. Scenario in the millimetre wavebands with $K=2$ and one small cell interferer (at a distance $R+d$ from the user equipment), where $d$ is the distance from the eNB to the user equipment.

\section{Performance Evaluation}

In order to study the variation of the CNIR and supported throughput with the coverage distance (for the downlink, DL), 
$R$, the supported throughput is computed through the implicit function analytical formulation that was already applied in [8], [9]. One example of the variation of the PHY throughput that corresponds to the curves of CNIR for the mmWaves is presented in Fig. 5, where sharing is considered with the interferer from MO \#2 placed at a distance $R+d$ from the UE.

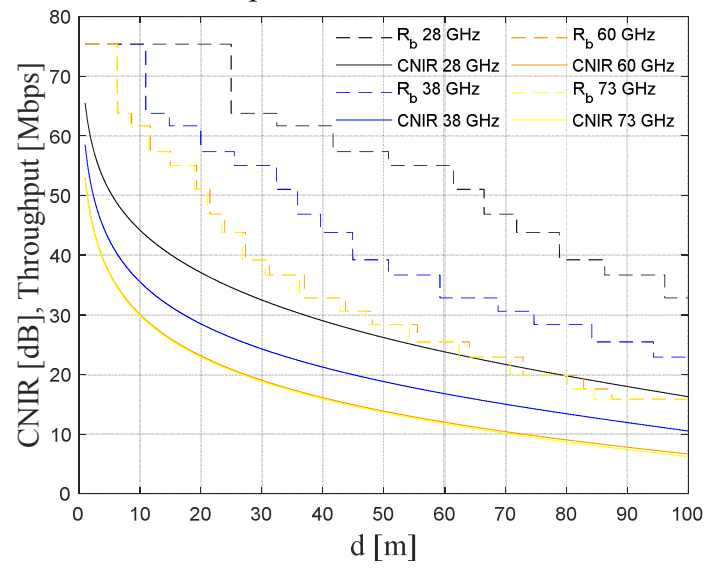

Fig. 5. Variation of the SINR and PHY throughput with $d$ for $28 \mathrm{GHz}, 38 \mathrm{GHz}$, $60 \mathrm{GHz}, 73 \mathrm{GHz}$, for $R=100 \mathrm{~m}$ and $\sigma \neq 0$.

Fig. 6 analyses the variation of the supported throughput for $K=3$, for the pico-cellular layer, $\mathrm{LoS}, \mathrm{BW}=10 \mathrm{MHz}$, and $f=2.6$ $\mathrm{GHz}$ and $3.5 \mathrm{GHz}$. The four relevant positions for the interferer are considered together with the case without interference (from the cell of $\mathrm{MO} \# 2$, considering the shared frequency band).

For a bandwidth of $20 \mathrm{MHz}$ the best cases have been considered, which correspond to the interferers placed at Northeast and East. The breakpoint distance is computed as follows:

$$
d_{B P}=\frac{4 h_{U} h_{B S} f}{3 * 10^{8}}
$$

where $f$ is the frequency in $(\mathrm{Hz}), h_{u}$ is the user height and $h_{B S}$ is the base station height.

By using (4), one concludes that the breakpoint distances for 2.6 GHz, 3.5 GHz, and mmWaves (i.e., 28, 38, 60 and $70 \mathrm{GHz}$ ), are $156,210,1680,2280,3600$ and $4380 \mathrm{~m}$, respectively.

All the parameters considered for the UHF/SHF bands from Table 1 are based on the values from [11].

By comparing all the curves in Fig. 6 we can observe that for intermedium values of the coverage distance the supported throughput at $2.6 \mathrm{GHz}$ is higher than at $3.5 \mathrm{GHz}$, because of the impact of the different values for the breakpoint distance. With sharing, in general, lower values of the system capacity are supported.

TABLE I. PARAMETERS CONSIDERED IN THE ANALYSIS (EXTRACTED FROM [11], [12])

\begin{tabular}{|l|c|c|}
\hline Parameter & UHF/SHF & mmWave \\
\hline Transmitter power & $-7 \mathrm{dBW}$ & $0 \mathrm{dBW}$ \\
\hline Transmitter gain & $17 \mathrm{dBi}$ & $3 \mathrm{dBi}$ \\
\hline Receiver gain & $0 \mathrm{dBi}$ & $0 \mathrm{dBi}$ \\
\hline Carrier & $20 \mathrm{MHz}$ & $20 \mathrm{MHz}$ \\
\hline Noise Figure & $5 \mathrm{~dB}$ & $7 \mathrm{~dB}$ \\
\hline Height (Base Station) & $9 \mathrm{~m}$ & $7 \mathrm{~m}$ \\
\hline Height (User Equipment) & $0.5 \mathrm{~m}$ & $1.5 \mathrm{~m}$ \\
\hline
\end{tabular}

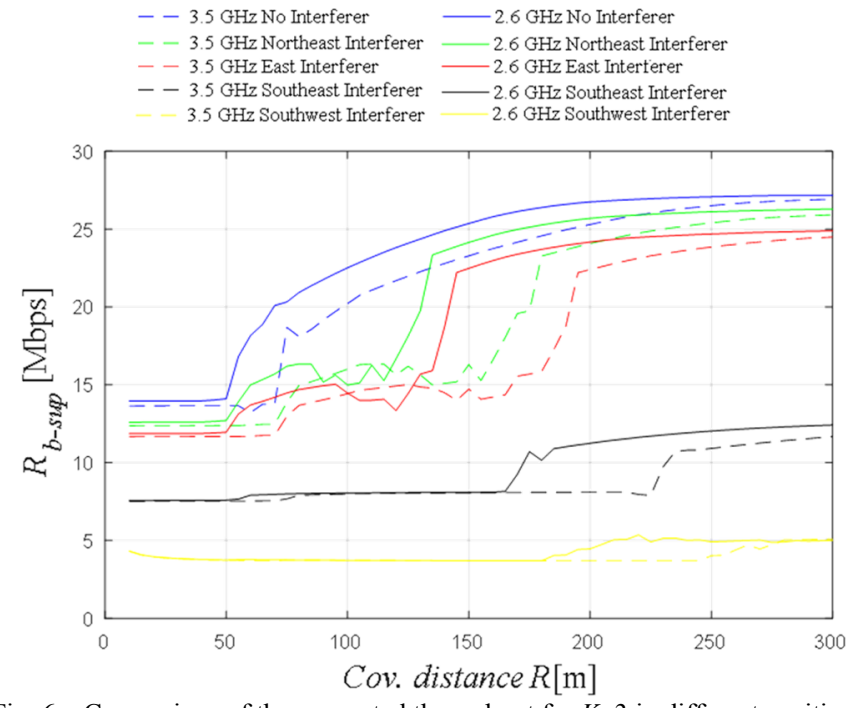

Fig. 6. Comparison of the supported throughput for $K=3$ in different positions of the Interferer from MO \#2, without interference, LoS, $B W=10 \mathrm{MHz}$

For the Southwest and Southeast interference scenarios, the supported throughput in both frequency bands is similar up to $R=200 \mathrm{~m}$ and $R=180 \mathrm{~m}$, respectively.

One considers that $R$ varies between 10 and $300 \mathrm{~m}$, and that $\sigma \neq 0$ for mmWaves [8]. By analysing the results from Fig. 7, generally, the supported throughput for the sharing scenario is higher for the $28 \mathrm{GHz}$ frequency band compared to the remaining millimetre frequency bands $(38,60$ and $73 \mathrm{GHz})$. One observes that the system capacity decreases for increasing coverage distances. In the sharing scenario at mmWaves, the supported throughput is highly reduced by the interference caused by the cell from a different mobile operator. However the ubiquitous coverage of the mobile communication system remains, as the PHY throughput does not reach zero inside the cell.

To understand the practicability of considering spectrum sharing in UHF, SHF and mmWaves, Fig. 8 puts together all the curves from the analysis for these frequency bands. On the sharing scenario, it is noticeable that, due to the behaviour arising from the two-slope model (Umi LoS) applied to 2.6 and $3.5 \mathrm{GHz}$, the supported throughput at the mmWave is higher than for the UHF/SHF bands for the shortest $R \mathrm{~s}$.

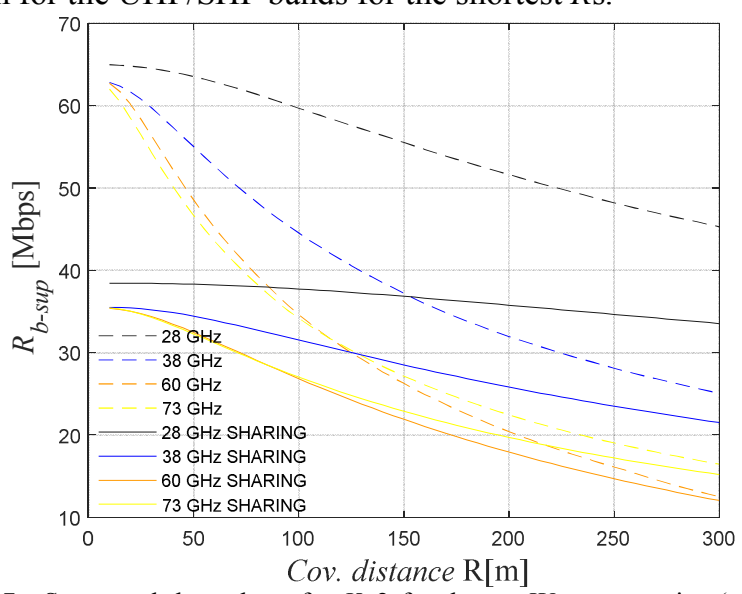

Fig. 7. Supported throughput for $K=2$ for the mmWave scenarios (with and without frequency sharing), with $B W=20 \mathrm{MHz}$. 
As an example, it is worthwhile to highlight that, with the Northeast Interferer, the supported throughput at 28, 38 and 60/73 GHz is higher than:

- the supported throughput at $2.6 \mathrm{GHz}$, for coverage distances up to circa 130,110 and $60 \mathrm{~m}$, respectively;

- the supported throughput at $3.5 \mathrm{GHz}$, for coverage distances up to circa 160, 95 and $75 \mathrm{~m}$, respectively.

For longer $R \mathrm{~s}$, the supported throughput from the sharing scenario converges to the one from the scenario without sharing.

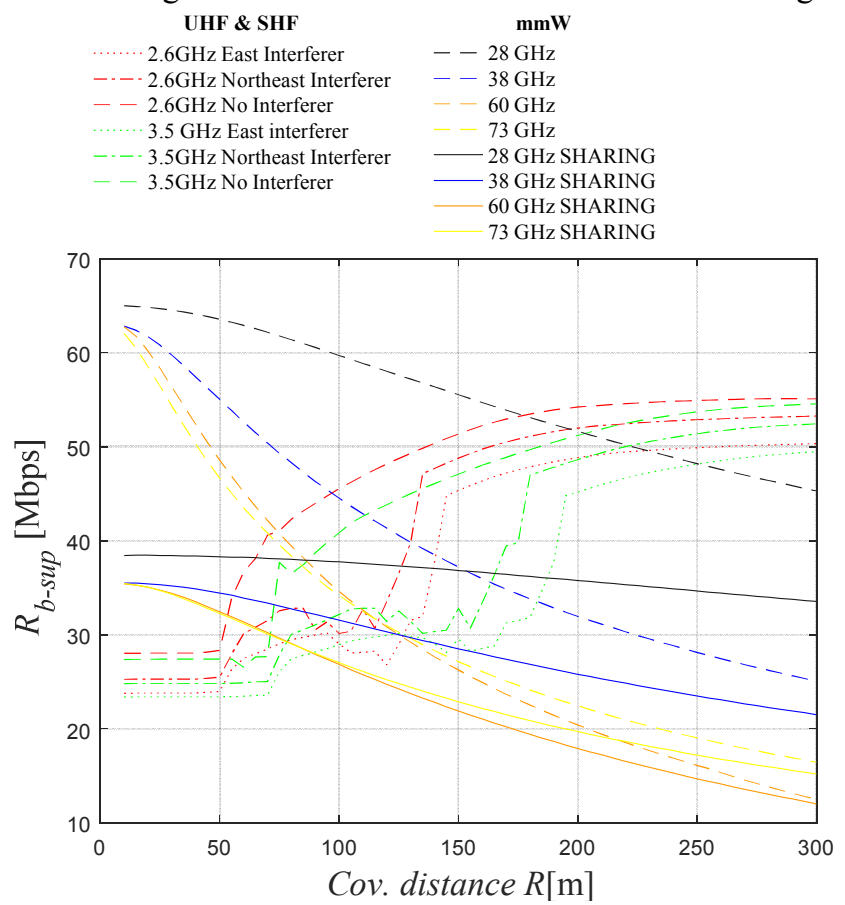

Fig. 8. Comparison of the supported throughput between UFH, SHF bands and millimetre wavebands, with $B W=20 \mathrm{MHz}$.

While considering spectrum sharing one assumes that the cell from $\mathrm{MO} \# 2$ can cause interference in the remaining co-channel cells from MO \#1 either in a permanent (traffic from users supported in the cell $100 \%$ of the time) or intermittent way (traffic supported only part of the time). One can define the percentage of spectrum sharing use, $\alpha$, meaning that with $\alpha=0$ there is no traffic usage in the cell from MO \#2 (shared spectrum), while for $\alpha=1$ resources are permanently occupied. By considering this very simple assumptions, Figs. 9, 10 and 11 shows the supported throughput for $0 \leq \alpha \leq 1$. It is observable that the surface represents values of $R_{b-\text { sup }}$ in between the scenarios without sharing $(\alpha=0)$ and $100 \%$ sharing $(\alpha=1)$.

In order to study the influence of different frequency bands in the results of the PHY throughput as a function of $R$, three dimensional (3D) graphs have been drawn, as in [8], [10], where the cell size $(R)$ varies from $10 \mathrm{~m}$ to $300 \mathrm{~m}$.

Through the analysis of $3 \mathrm{D}$ view graphs of the PHY throughput, e.g., the Northeast interferer in Fig. 12 (the best case for the considered sharing scenarios), the higher supported throughput has an increasing behaviour when the coverage reaches circa 140-150 m, around the breakpoint distance $\left(d_{B P}\right)$. After this distance, the highest MCSs are supported for a larger region of the cell and then the supported throughput is continuously increasing (in the range of $R$ s presented here).

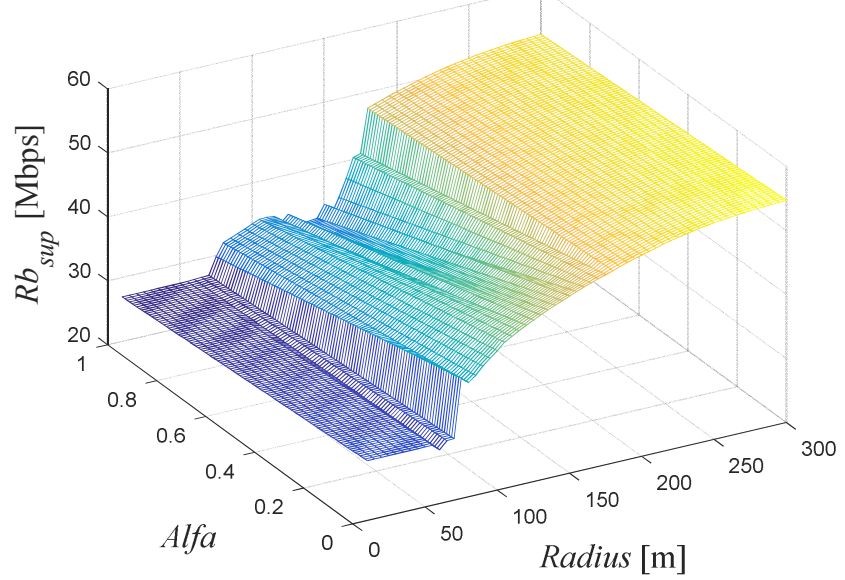

Fig. 9. Variation of the supported throughput for different percentages of spectrum sharing use, $\alpha(0 \leq \alpha \leq 1)$, for $f=3.5 \mathrm{GHz}$.

Supported throughput for different percentages of spectrum sharing use, $28 \mathrm{GHz}$

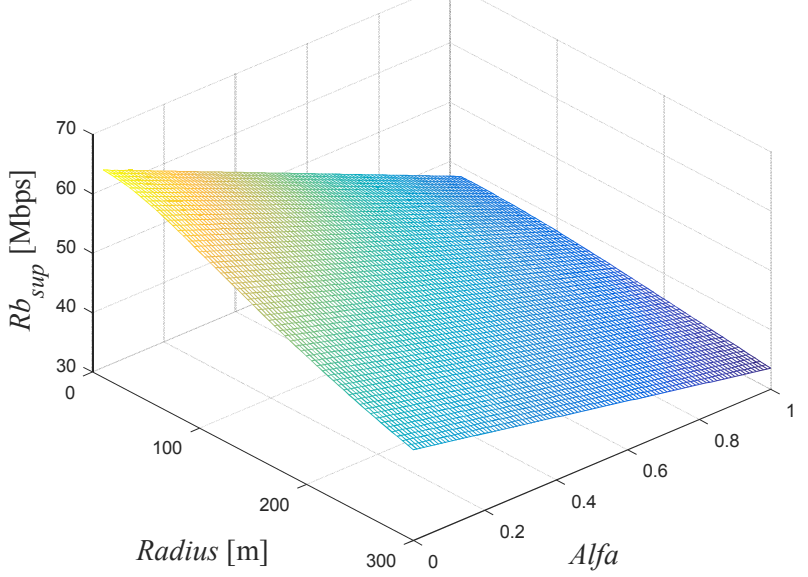

Fig. 10. Variation of the supported throughput for different percentages of spectrum sharing use, $\alpha(0 \leq \alpha \leq 1)$, for $f=28 \mathrm{GHz}$.

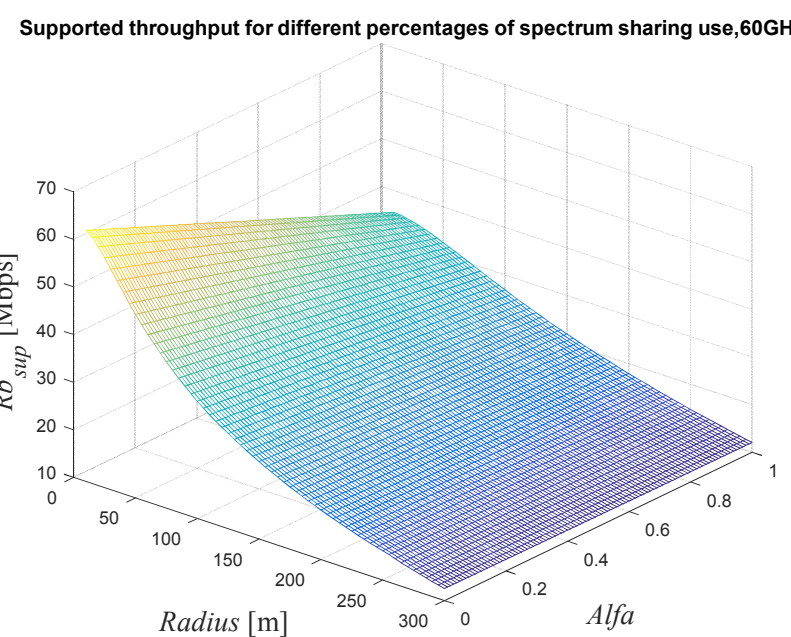

Fig. 11. Variation of the supported throughput for different percentages of spectrum sharing use, $\alpha(0 \leq \alpha \leq 1)$, for $f=60 \mathrm{GHz}$.

In the $3 \mathrm{D}$ view chart for the scenario without sharing from Fig. 13, the higher PHY throughput is also reached for long distances, and the change of behaviour occurs for distances of circa $d_{B P} / r_{c c}$, as discussed in [10]. 


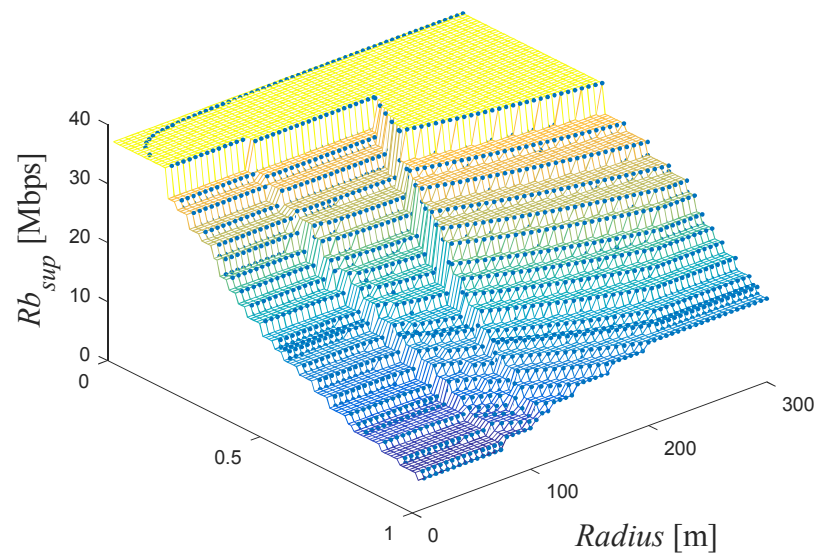

Normalized Distance

Fig. 12. 3D view graph for the supported throughput mapped into MCSs for the $3.5 \mathrm{GHz}$ with Northeast Interferer and $R=300 \mathrm{~m}$.

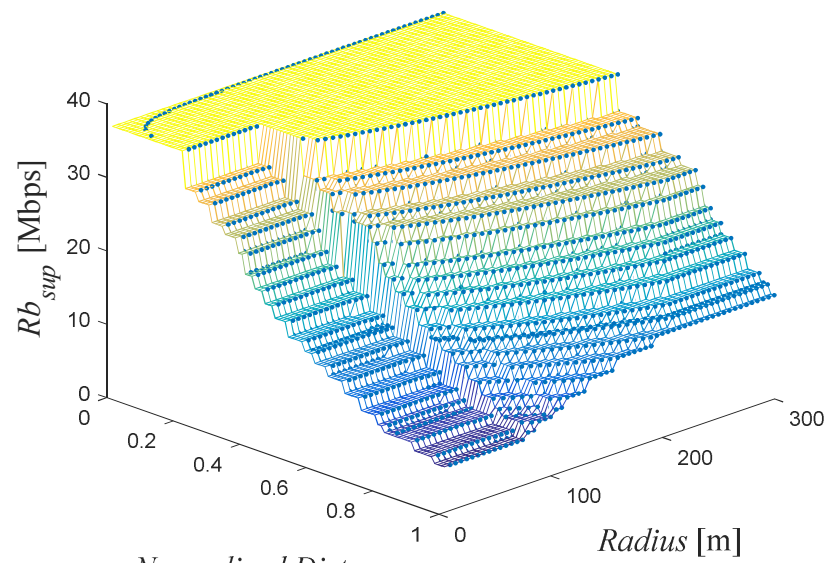

Normalized Distance

Fig. 13. 3D view graph for the supported throughput mapped into MCSs for the $3.5 \mathrm{GHz}$ without sharing and $R=300 \mathrm{~m}$.

In the mmWaves, an example is presented in Fig. 14 for the PHY throughput at $28 \mathrm{GHz}$. As the breakpoint distance is a 1680 $\mathrm{m}$, the behaviour is the one for a single slope propagation model.

As the coverage distances increases the upper MCSs are supported up to shorter distances inside the cells. These results allows for interpreting why at the mmWaves the system capacity is clearly higher for the shortest coverage distances, ceasing to be better for longer distances.

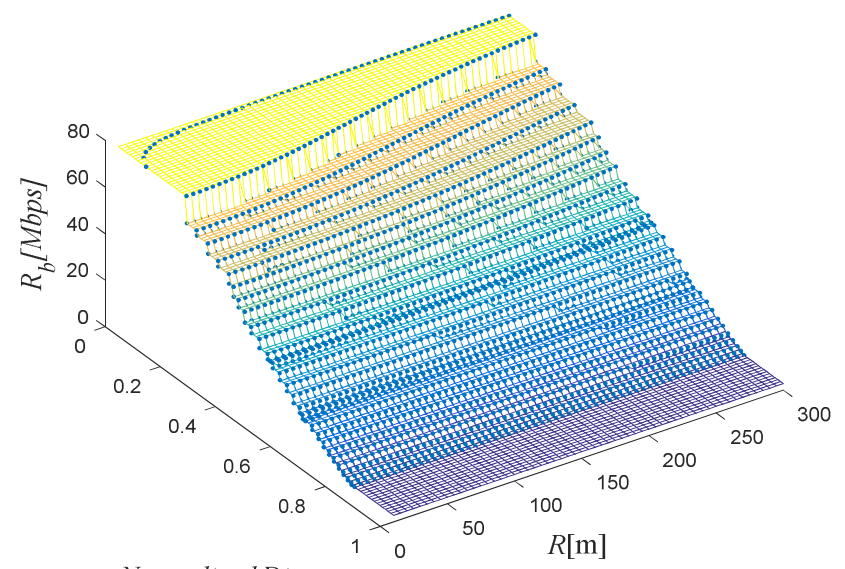

Normalized Distance

Fig. 14. 3D view graph for the supported throughput mapped into MCSs for the $28 \mathrm{GHz}$ with Interferer and $R=300 \mathrm{~m}$

\section{CONCLUSION}

This study analyses the viability of spectrum sharing for Ultra High Frequencies and Super High Frequencies as well as for the mmWaves. The PHY and equivalent supported throughput within cells have been assessed while considering reuse pattern $K=3$ and $K=2$ for UHF/SHF and mmWaves, respectively. We assume in this preliminary phase that LTE is also considered for the mmWaves. The computation of interference considers LoS propagation models. From this preliminary analysis, we have learned that the highest system capacity and the highest modulation and coding schemes are achievable for the shortest coverage distances at mmWaves whereas the supported throughput for long coverage distances is clearly more favourable for UHF/SHF with/without sharing.

In fact, due to the behaviour arising from the two-slope propagation model (Umi LoS) applied to 2.6 and $3.5 \mathrm{GHz}$, the supported throughput at the mmWaves is higher than the one for the UHF/SHF bands for the shortest $R \mathrm{~s}$.

\section{ACKNOWLEDGMENT}

Authors would like to thank Professor Jon Peha, Eng. Rui Paulo and Eng. Rooderson Andrade for the fruitful discussions.

\section{REFERENCES}

[1] A. Checko et al., "Cloud RAN for Mobile Networks-A Technology Overview, " in IEEE Communications Surveys \& Tutorials, vol. 17, no. 1, pp. 405-426, Firstquarter 2015

[2] 5G Americas, 2016.[Online]. Available:http://www.5gamericas.org/en/ (accessed on 19 January 2018).

[3] J. Mcmenamy, I. Macaluse, N. Marchetti, L. Doyle, "A Methodology to Help Operators Share the Spectrum through an Enhanced Form of Carrier Aggregation," in IEEE International Symposium on Dynamic Spectrum Access Networks (DYSPAN), pp. 334-345, Mclean, VA, April 2014.

[4] J. M. Peha, "Sharing Spectrum Through Spectrum policy Reform and Cognitive Radio," in Proceedings of the IEEE, vol.91, no. 4, pp. 708-719, April 2009.

[5] S. Tang and B. L. Mark, "Performance Analysis of a Wireless Network with Opportunistic Spectrum Sharing," in IEEE Global Telecommunications Conference, pp. 4636-4640, Washington, DC, USA, Nov. 2007.

[6] 3GPP TR 36.889 V1.0.1, "Feasibility Study on License-Assisted Access to Unlicensed Spectrum," June 2015.

[7] S. Y. Lien, C. C. Chien, H. L. Tsai, Y. C. Liang and D. I. Kim, "Configurable 3GPP Licensed Assisted Access to Unlicensed Spectrum," in IEEE Wireless Communications, vol.23, no. 6, pp. 32-39, Dec. 2016.

[8] E. Teixeira, F. J. Velez, "Extending the LTE-Sim Simulator with MultiBand Scheduling Algorithms for Carrier Aggregation in LTE-Advanced Scenarios," 2018 IEEE 87th Vehicular Technology Conference (VTC Spring), Porto, 2018.

[9] F. J. Velez, D. Robalo and J. A. Flores, "LTE radio and network planning: Basic coverage and interference constraints," 2015 7th IEEE LatinAmerican Conference on Communications (LATINCOM), Arequipa, 2015 , pp. 1-6.

[10] S. Sousa, F. J. Velez and J. M. Peha, "Impact of propagation model on capacity in small-cell networks, " 2017 International Symposium on Performance Evaluation of Computer and Telecommunication Systems (SPECTS), Seattle, WA, 2017, pp. 1-8.

[11] Guidelines for evaluation of radio interface technologies for IMTAdvanced,ReportITU-R,M.1255,1997 (https://www.itu.int/dms_pubrec/itu-r/rec/m/R-REC-M.1225-0-199702I!!PDF-E.pdf))

[12] G. R. Maccartney, T. S. Rappaport, M. K. Samimi and S. Sun, "Millimeter-Wave Omnidirectional Path Loss Data for Small Cell 5G Channel Modeling," in IEEE Access, vol. 3, pp. 1573-1580, 2015. 